خليل: دراسة مختبرية لتأثير موقع وارتفاع هدار غاطس في قناة رئيسة على الجريان في قناة فرعية

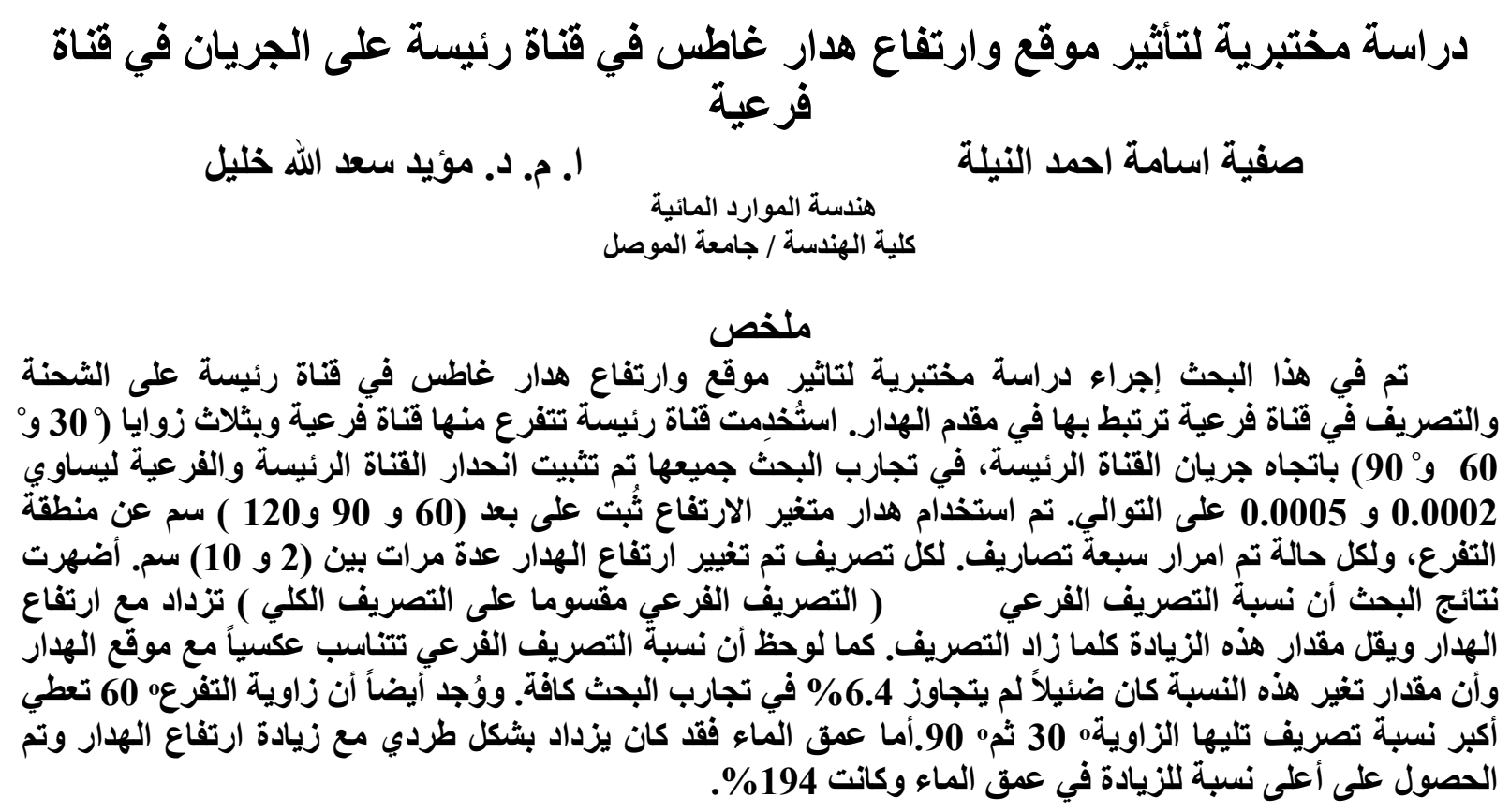

\title{
Laboratory Study of Weir Height and Location Effect in a Main Channel on the Branching Channel Flow
}

\author{
Safiya Usama Ahmed Al-Neelah Moayad Sa'ad Allah Khaleel \\ Water Resources Engineering \\ College of Engineer / Mosul University
}

\begin{abstract}
In this paper an experimental work was conducted to study the effect of a weir height and location in a main channel on the head and discharge of branching channel connected upstream the weir, with three different angles $\left(30^{\circ}, 60^{\circ}\right.$ and 90 ). The bed slope of the main and branch channel was kept constant and it was equal to 0.0002 and 0.0005 , respectively. A variable height weir was fixed in the main channel at different distance $(60,90$ and $120 \mathrm{~cm})$ downstream the branching point. For each location, seven different discharges were used, and for each discharge, the height of the weir was changed from 2 to $10 \mathrm{~cm}$. The results of the research showed an existence branching and main discharges, and a similar relation between branch discharge and the weir height. It is also found that the ratio of discharge (the branch discharge divided by main discharge) increases with increasing weir height, and the magnitude of this decreases as the discharge increases. The result also showed that the angle 60 of branch channel gave highest values for discharge ratio. The depth of water in the branch channel varies directly with the height of weir, and a maximum percentage of increases of depth observed in all experiments were $194 \%$.
\end{abstract}




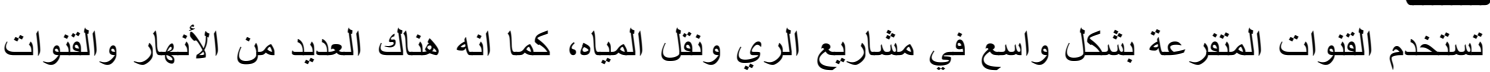

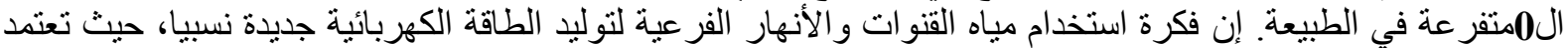

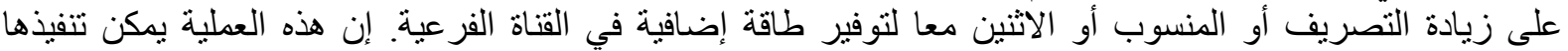

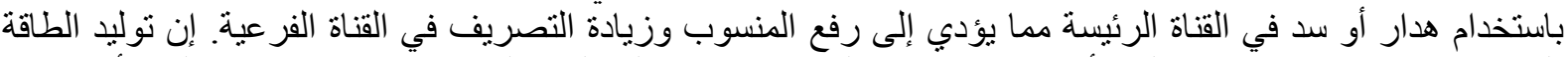

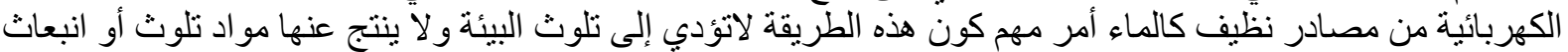

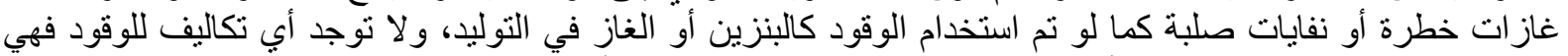

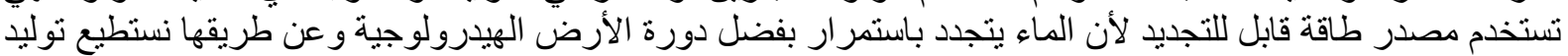

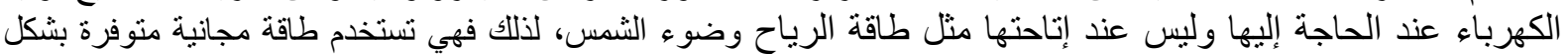

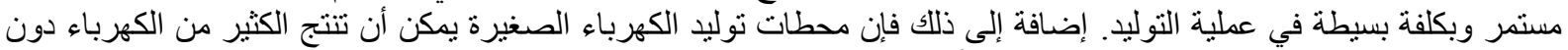

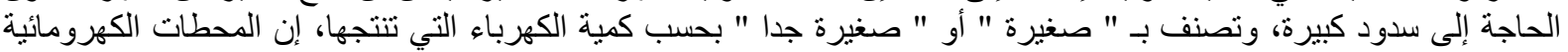

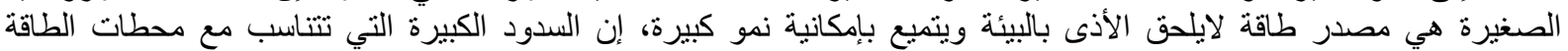

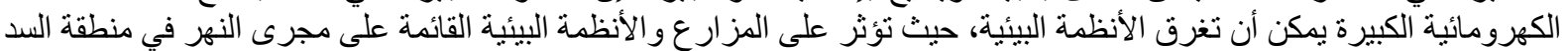

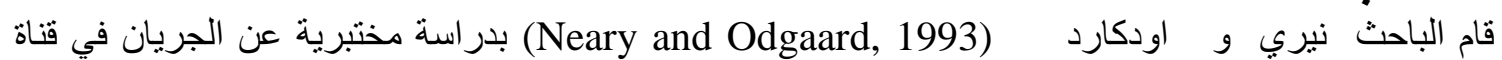
مفتوحة متفرعة بزاوية 90. تم صنع القناتين الرئيسة والفرعية من الفاييركلاس وبقعر ناعم للقناة الفرعية، أما في القناة

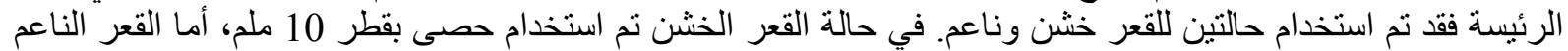

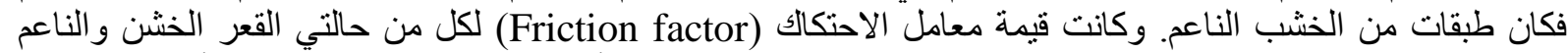

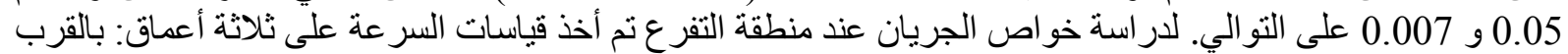

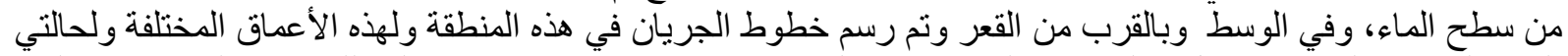

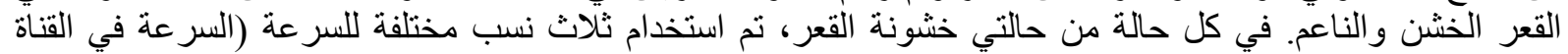

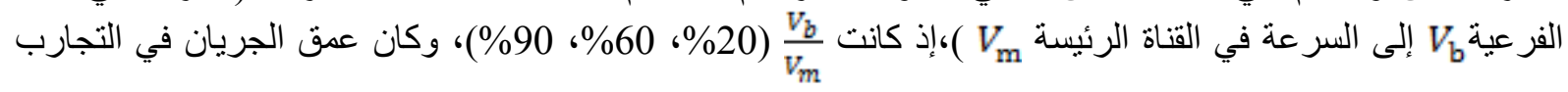

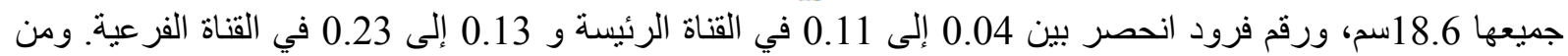

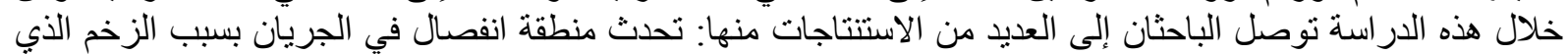

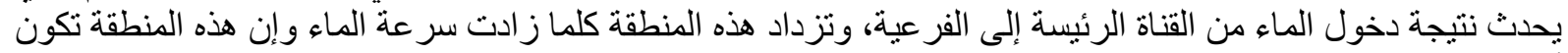

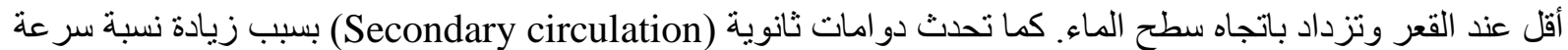

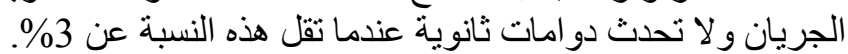

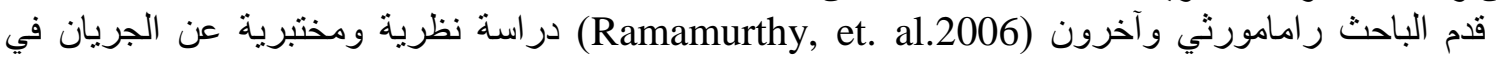

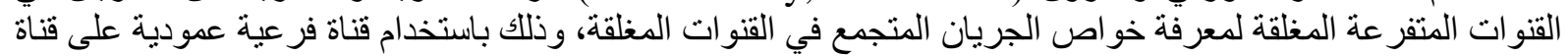

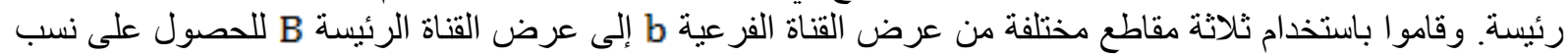

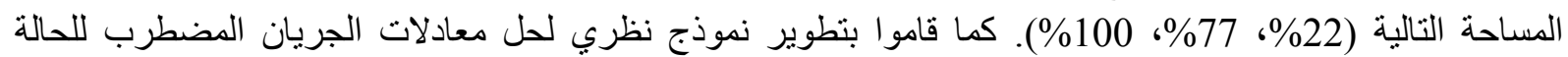

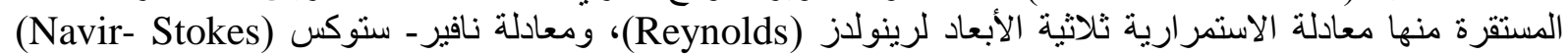

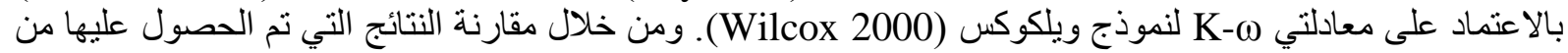

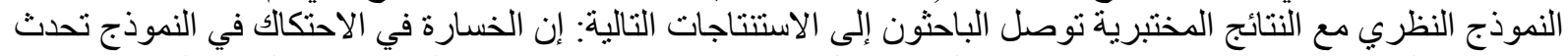

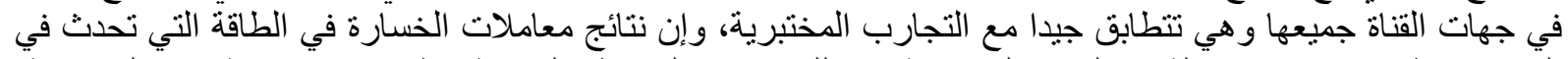

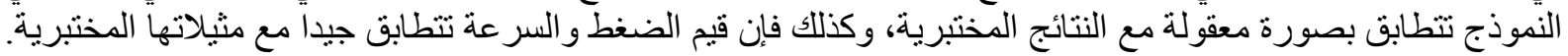

كما لاحظو ا إن منطقة الانفصال التي حدثت في النموذج تنطابق النقان مع منطقة الانفصال التي حدثت في القناة المختبرية.

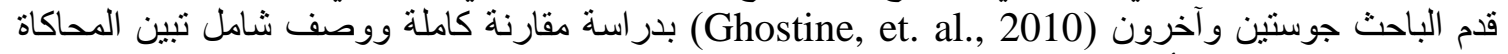

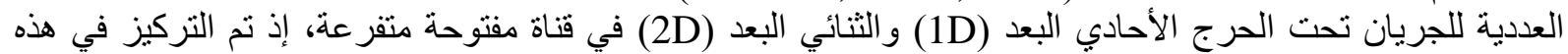

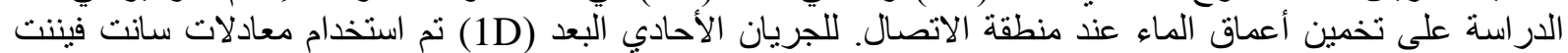

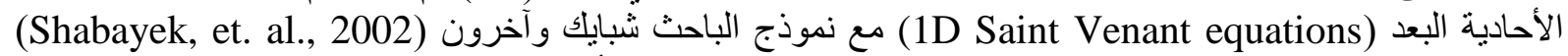

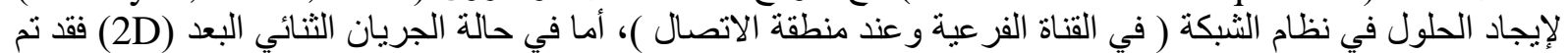

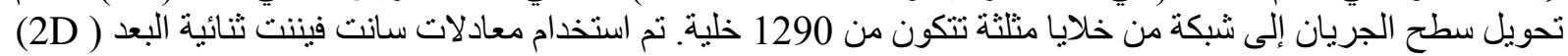

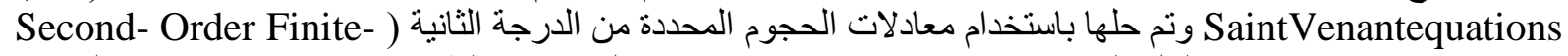
(Volume method 
المختبرية لتايلور(Taylor,1944) و ويير وكريند (Webber and Greated, 1966) وجيرام (Gurram, 1994)

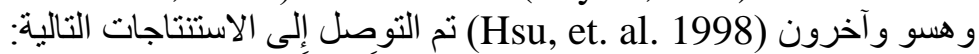

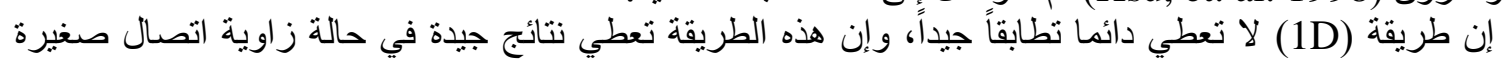

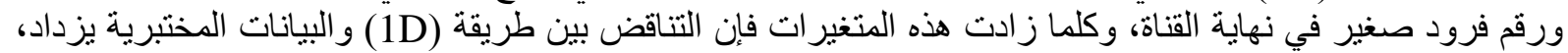
بينما طريقة (2D) تتطابق جيدا مع البيانات المقاسة. وبذللك توصلو التية إلى أن معادلات سانت فينتانت أحادية البعد تطبق في

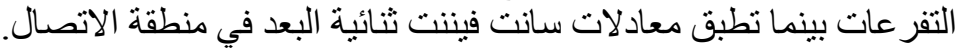

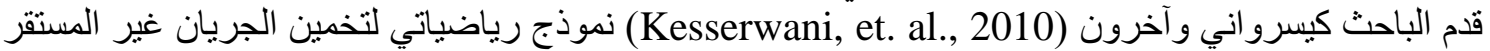

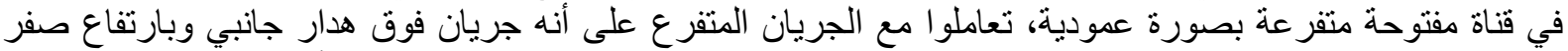

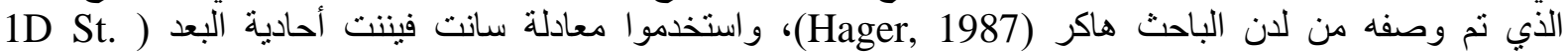
(Venantequation

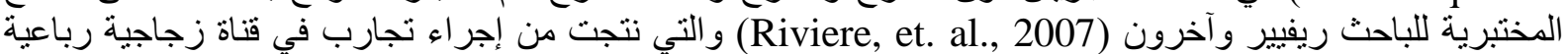

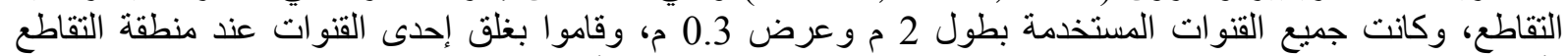

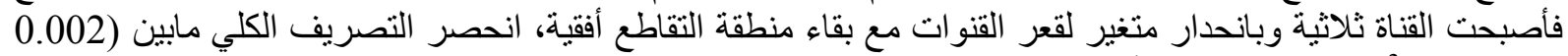

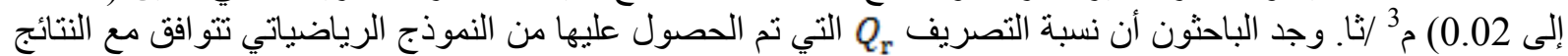

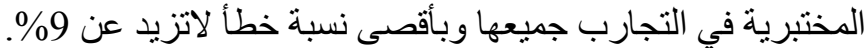

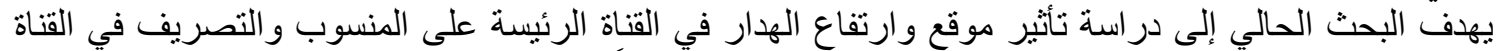

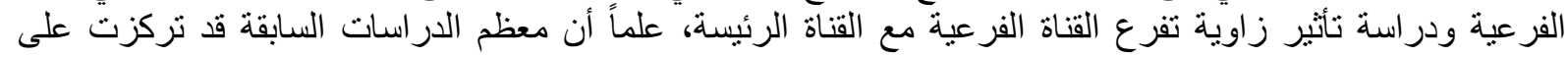

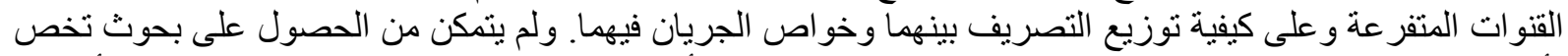

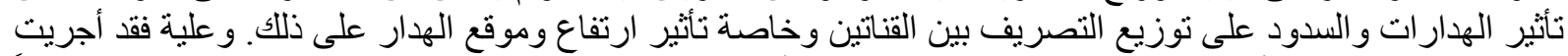

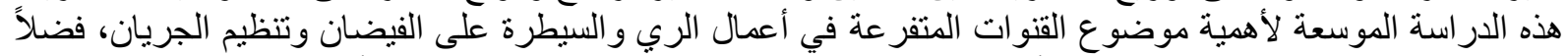

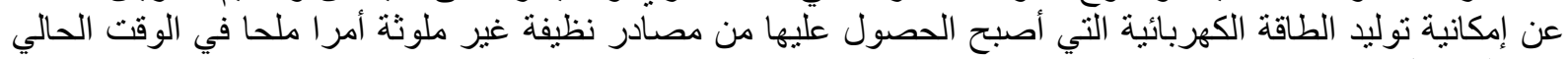

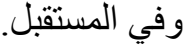

\section{التجارب المختبرية}

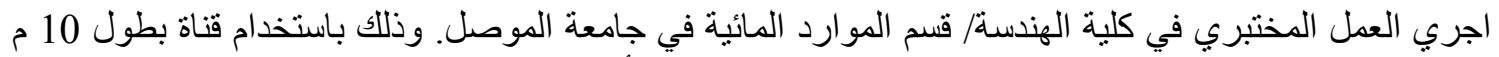

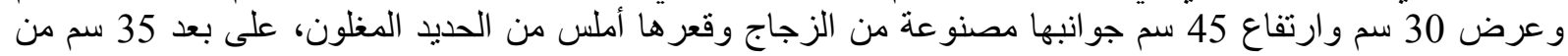

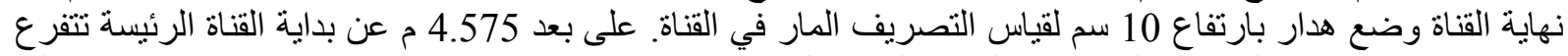

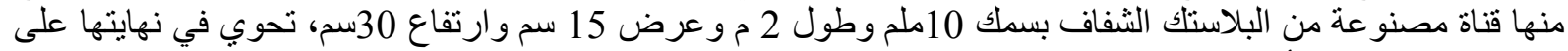

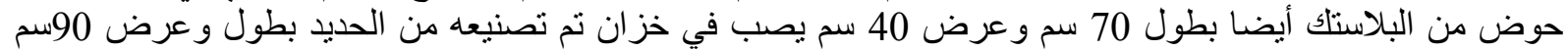

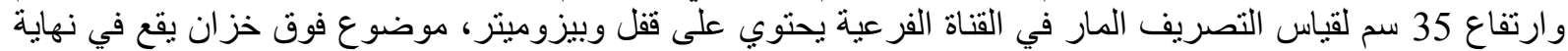

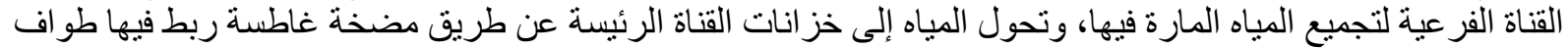

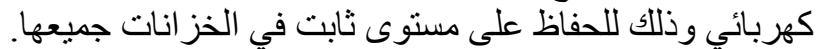

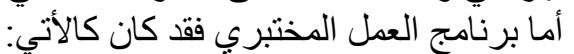

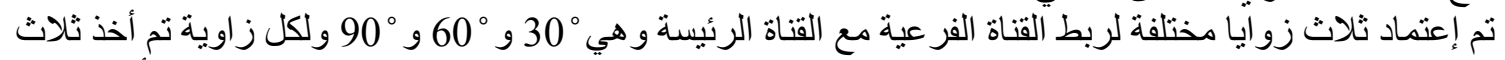

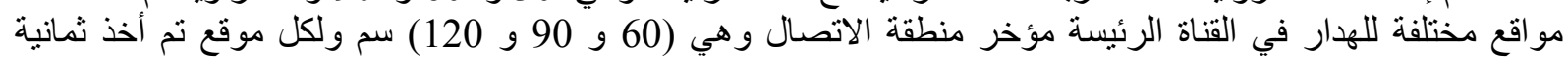

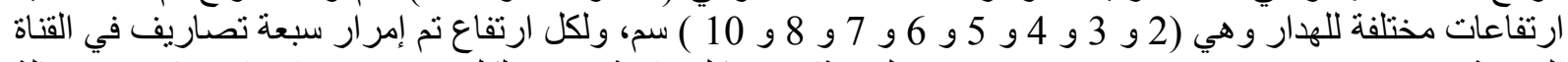

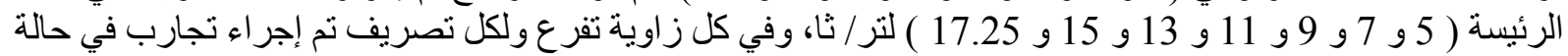

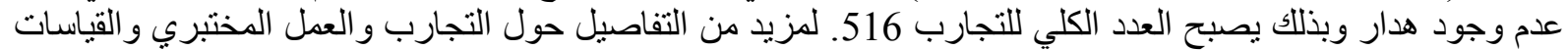

يمكن مر اجعة المصدر (النيلة، 2011).

\section{تحليل النتائج المختبرية \\ إن الهدف من هذا التحليل هو التركيز على تاثير الهدار على التصريف في القناة الفرعية وعمق الجريان فيهاو إيجاد مدى هذا التأثير.

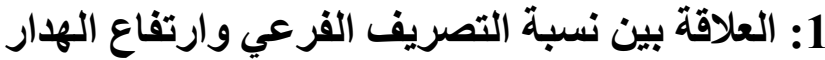

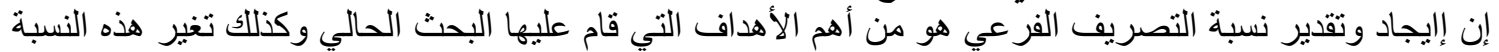

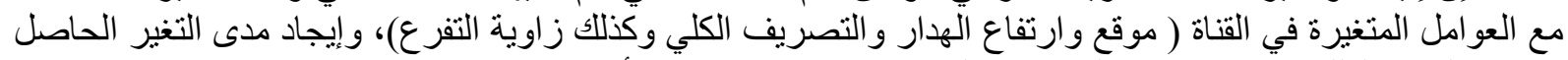

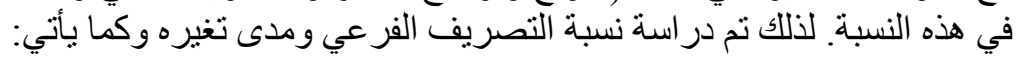




\section{1-1: تغير نسبة التصريف الفرعي مع ارتفاع الهار والتصريف الكلي وبقاء موقع الهار وزاوية}

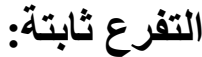

تم رسم تغير قيم نسبة التصريف الفرعي Qr مع ارتفاع الهدار P لكل تصريف من التصاريف السبعة للقناة الرئيسة

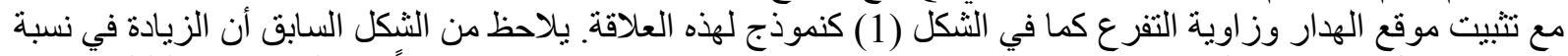

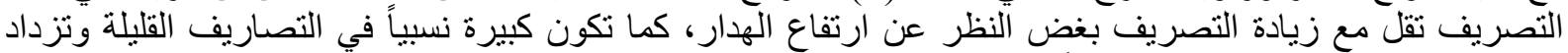

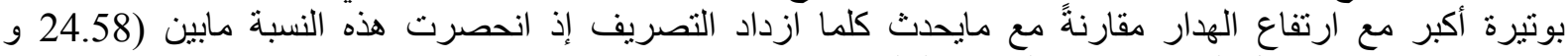
$\mathrm{Q}_{\mathrm{r}}=\mathrm{a}_{1}+\mathrm{a}_{2} \mathrm{P}$ 98.57)\% \% ويمكن تمثيل العلاقة بينهما بالصيغة التالية: إذ انحصرت معاملات التحديد R مابين (0.993 و 0.999). و الجدول (1) يبين قيم a و a الهدار المختلفة و (

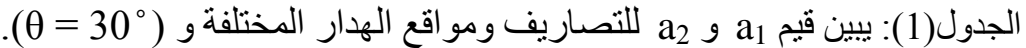

\begin{tabular}{|c|c|c|c|c|c|c|}
\hline \multirow{2}{*}{$\begin{array}{c}\mathbf{Q}_{\text {T }} \\
\mathbf{L} / \mathbf{s e c}\end{array}$} & \multicolumn{9}{|c|}{$\mathbf{6 0} \mathbf{~ c m}$} & \multicolumn{2}{c|}{$\mathbf{9 0} \mathbf{c m}$} & \multicolumn{2}{c|}{$\mathbf{1 2 0} \mathbf{~ c m}$} \\
\cline { 2 - 7 } & $\mathbf{a}_{\mathbf{1}}$ & $\mathbf{a}_{\mathbf{2}}$ & $\mathbf{a}_{\mathbf{1}}$ & $\mathbf{a}_{\mathbf{2}}$ & $\mathbf{a}_{\mathbf{1}}$ & $\mathbf{a}_{\mathbf{2}}$ \\
\hline $\mathbf{5}$ & 20.83 & 9.434 & 20.5 & 9.259 & 20.693 & 8.772 \\
\hline $\mathbf{7}$ & 20.008 & 7.874 & 19.414 & 9.434 & 18.97 & 7.519 \\
\hline $\mathbf{9}$ & 19.649 & 6.757 & 19.616 & 6.623 & 19.286 & 6.494 \\
\hline $\mathbf{1 1}$ & 18.788 & 6.061 & 18.333 & 5.952 & 18.041 & 5.848 \\
\hline $\mathbf{1 3}$ & 18.3 & 5.556 & 18.104 & 5.495 & 17.946 & 5.376 \\
\hline $\mathbf{1 5}$ & 17.812 & 5.208 & 17.894 & 5.051 & 17.397 & 5.051 \\
\hline $\mathbf{1 7 . 2 5}$ & 16.833 & 4.785 & 16.869 & 4.695 & 16.885 & 4.608 \\
\hline
\end{tabular}

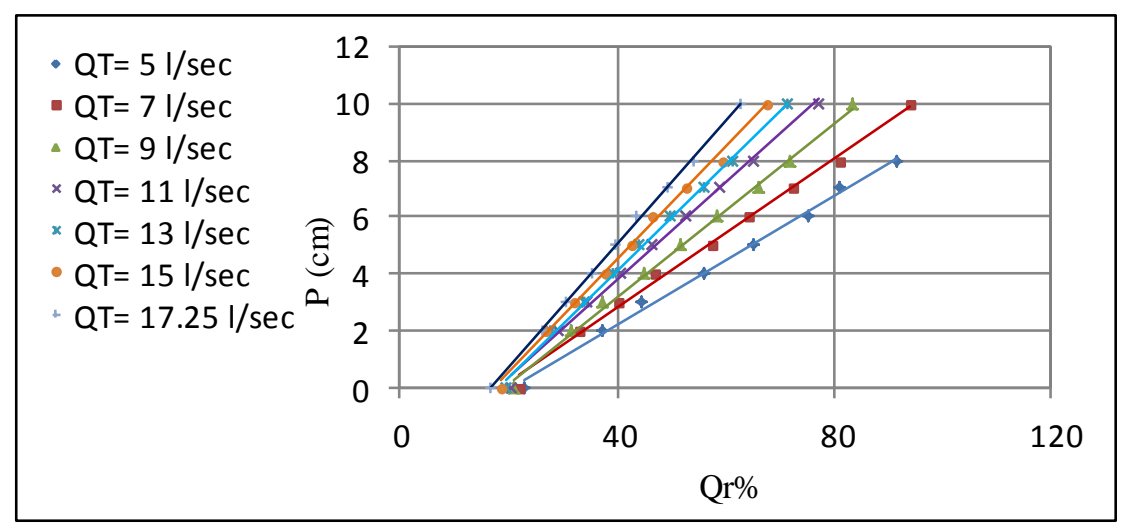

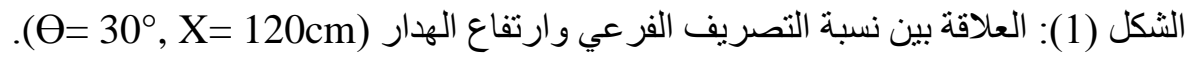

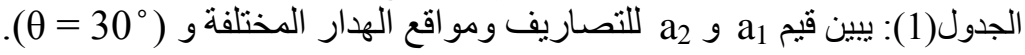

\section{2-1 تغير نسبة التصريف الفرعي مع ارتفاع الهارار وموقع الهار وبقاء التصريف وزاوية التفرع}

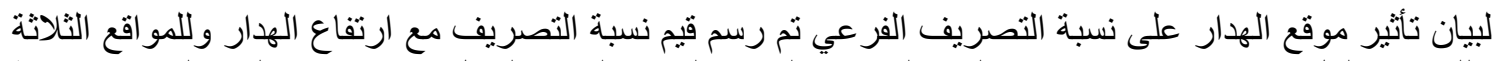

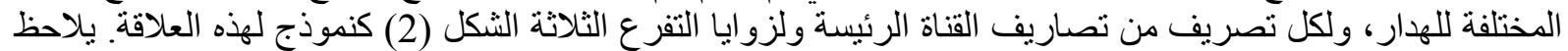

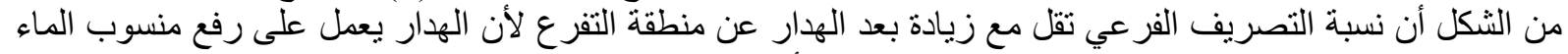

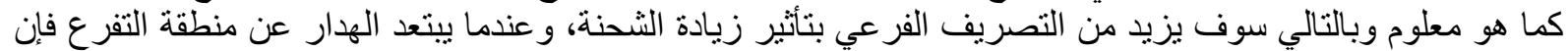

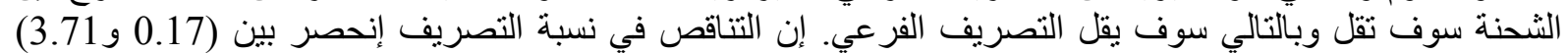

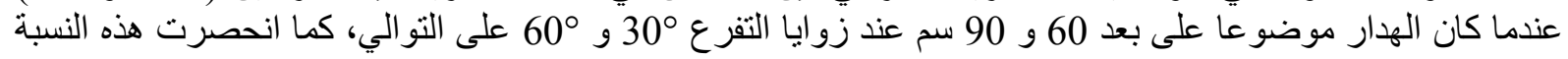




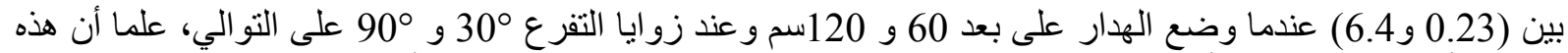

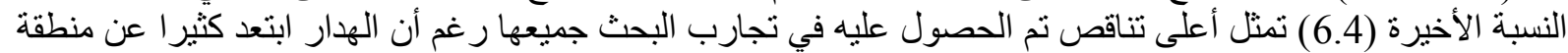

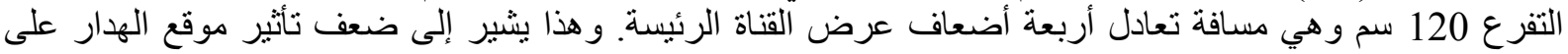
نسبة التصريف ضمن ظروف ومحددات هذا البحث.

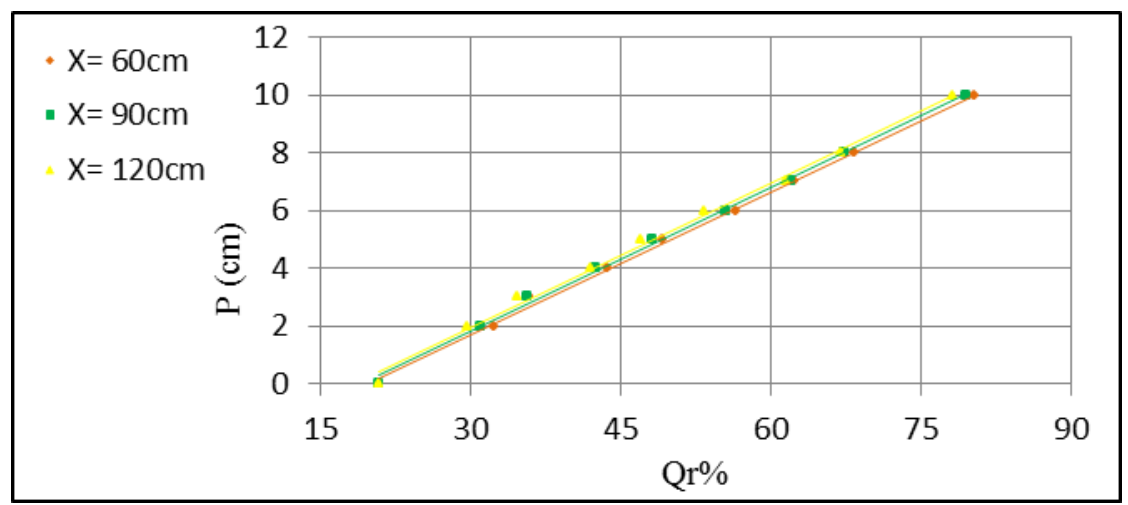

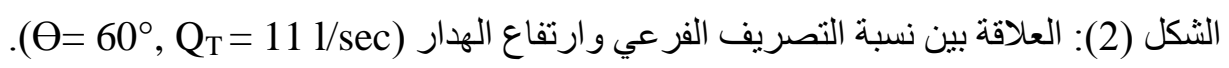

\section{3-1 :تغير نسبة التصريف الفرعي مع ارتفاع الهار وزاوية التفرع ويقاء التصريف وموقع الهار}

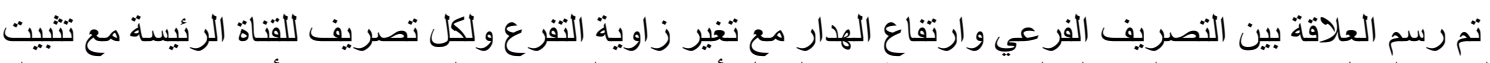

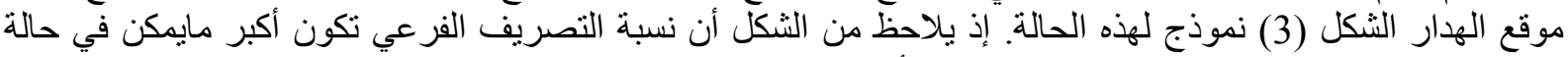

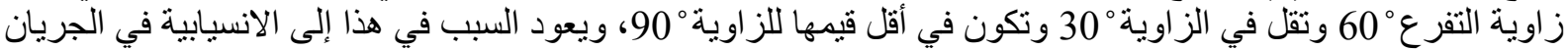

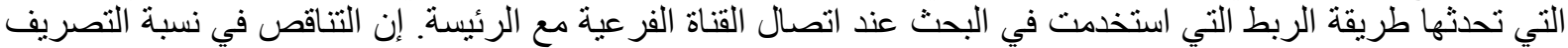

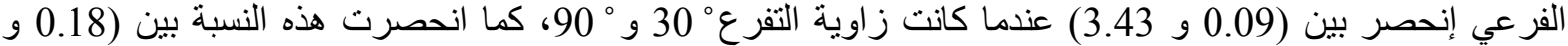
5.44) عندما كانت زاوية التفرع م60 و 9 90 ـ و هذه النسبة تؤشر أيضا التأثثر البسيط لز اوية التفرع على نسبة التصريف

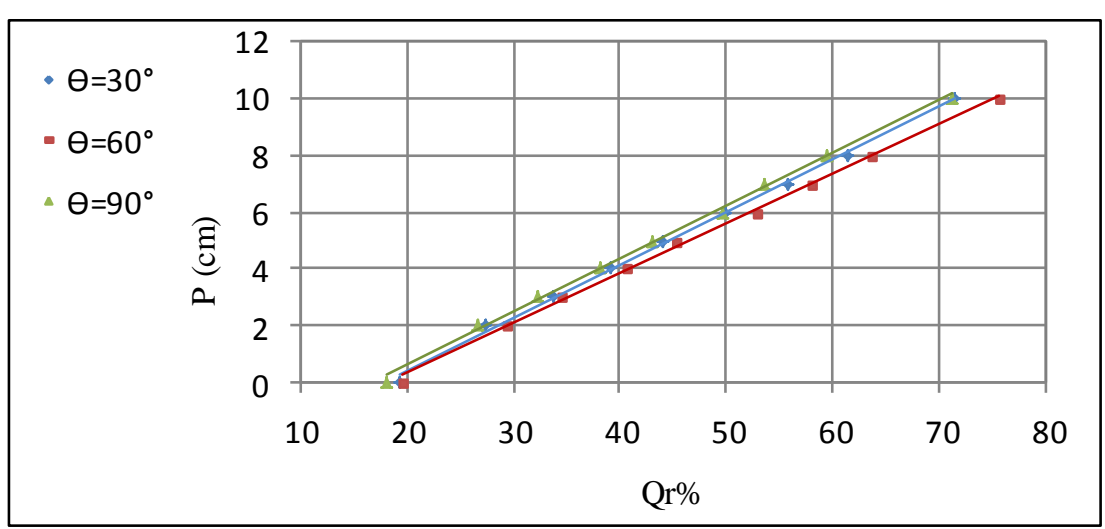

الفرعي.

الثكل (3): العلاقة بين نسبة التصريف الفرعي وارتفاع الهدار (X=120cm, QT = 13 1/sec).

2: تأثير الهار على عمق الجريان في القناة الفرعية:

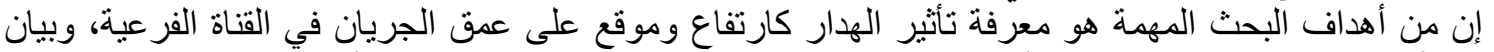
مدى هذا التأثير و الذي يبين مقدار الزيادة أو النقصان في شحنة الجريان في هذه القناة وهو الأمر المهم هنا. و وعليه فقد تم

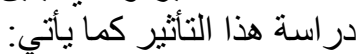


1-2: العلاقة بين عمق الجريان وارتفاع الهيار مع التصريف وبقاء زاوية التفرع وبعد الهارار ثابتاً:

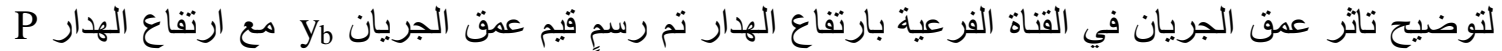

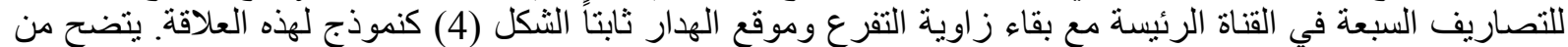
الثكل أن هناك علاقة طردية خطية بين المتغيرين و إن نسبة الزيادة في عمق الجريان العيان انحصرت بين (9.44 و 194.62) \% و ويكن تمثيل العلاقة بينهما بالصيغة التالية:

$\mathrm{y}_{\mathrm{b}}=\mathrm{a}_{3}+\mathrm{a}_{4} \mathrm{P}$

و انحصر معامل التحديد Re بين (0.981 و 0.999). الجدول (2) يبين قيم a و a للتصاريف ومواقع الهدار المختلفة و ( )

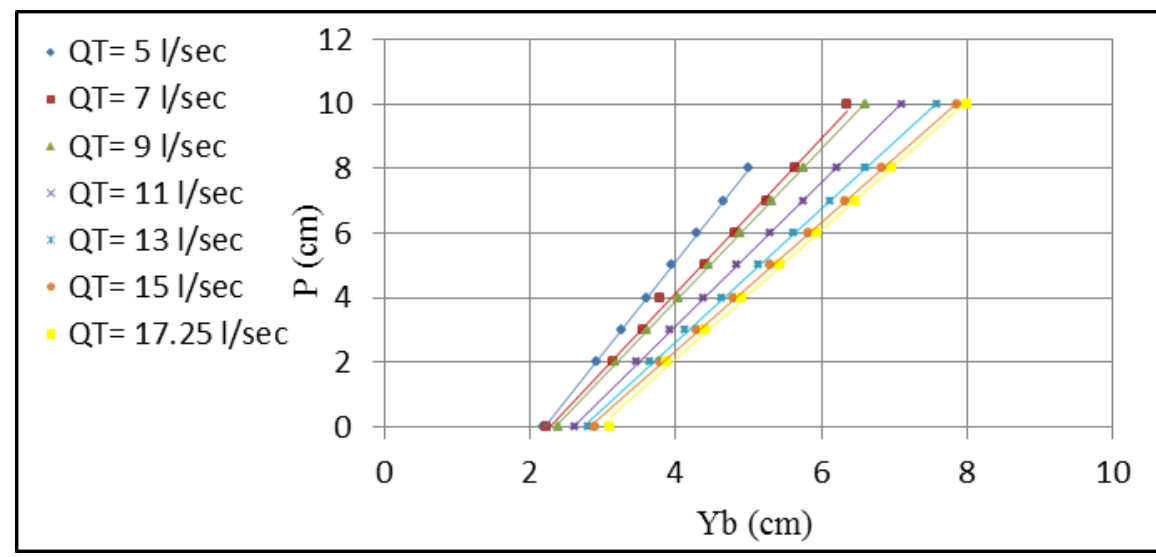

الشكل (4): العلاقة بين عمق الجريان الفر عي وارتفاع الهدار (O=90 , X=90cm). الجدول(2): يبين قيم a و a $a_{4}$ للتصاريف ومو اقع الهدار المختلفة و (

2-2: العلاقة بين عمق الجريان وارتفاع الهارار مع موقع الهدار ويقاء التصريف وزاوية التفرع ثابتة:

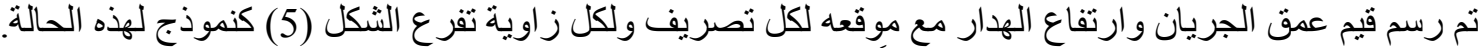

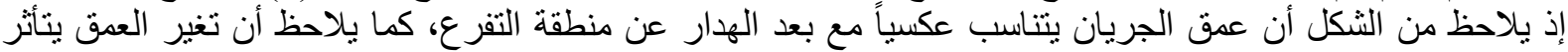

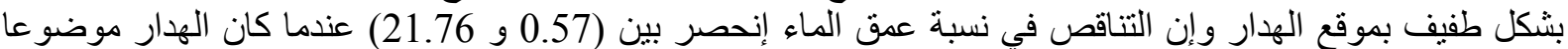

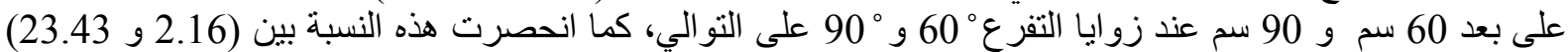

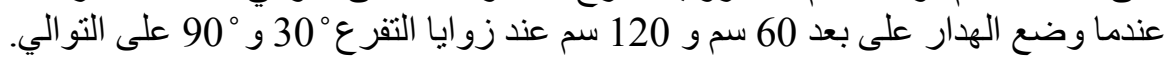

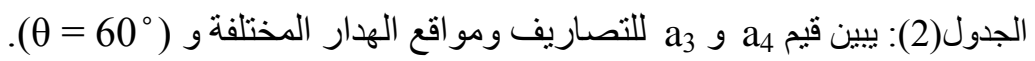

\begin{tabular}{|c|c|c|c|c|c|c|}
\hline \multirow{2}{*}{$\begin{array}{c}\mathbf{Q}_{\mathbf{T}} \mathbf{L} / \mathbf{s e c} \\
\end{array}$} & \multicolumn{2}{|c|}{$\mathbf{6 0} \mathbf{~ c m}$} & \multicolumn{2}{c|}{$\mathbf{9 0} \mathbf{~ c m}$} & \multicolumn{2}{c|}{$\mathbf{1 2 0} \mathbf{~ c m}$} \\
\cline { 2 - 7 } & $\mathbf{a}_{\mathbf{1}}$ & $\mathbf{a}_{\mathbf{2}}$ & $\mathbf{a}_{\mathbf{1}}$ & $\mathbf{a}_{\mathbf{2}}$ & $\mathbf{a}_{\mathbf{1}}$ & $\mathbf{a}_{\mathbf{2}}$ \\
\hline $\mathbf{5}$ & 2.474 & 0.473 & 2.465 & 0.461 & 2.428 & 0.447 \\
\hline $\mathbf{7}$ & 2.564 & 0.5 & 2.564 & 0.487 & 2.554 & 0.478 \\
\hline $\mathbf{9}$ & 2.878 & 0.48 & 2.867 & 0.469 & 2.836 & 0.461 \\
\hline $\mathbf{1 1}$ & 2.998 & 0.49 & 2.974 & 0.483 & 2.958 & 0.477 \\
\hline $\mathbf{1 3}$ & 3.533 & 0.464 & 3.529 & 0.46 & 3.491 & 0.458 \\
\hline $\mathbf{1 5}$ & 3.718 & 0.478 & 3.711 & 0.469 & 3.665 & 0.468 \\
\hline $\mathbf{1 7 . 2 5}$ & 4.239 & 0.535 & 4.159 & 0.532 & 4.132 & 0.529 \\
\hline
\end{tabular}


خليل: دراسة مختبرية لتأثير موقع وارتفاع هدار غاطس في قناة رئيسة على الجريان في قناة فرعية

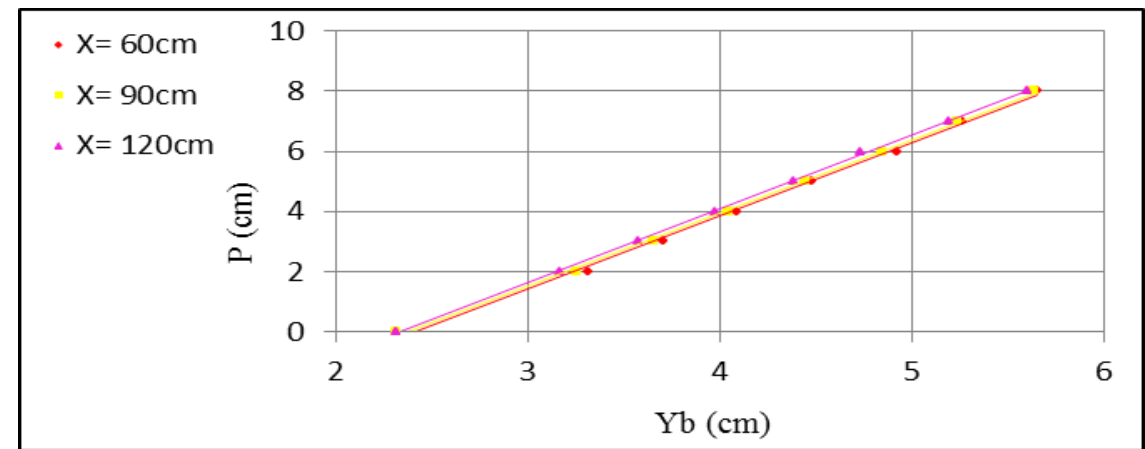

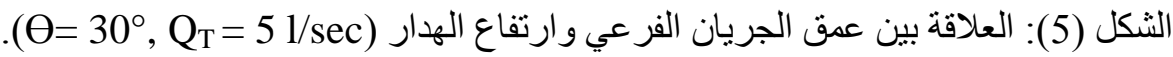

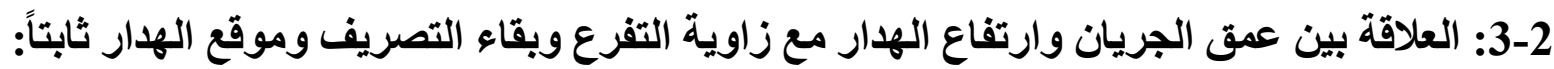

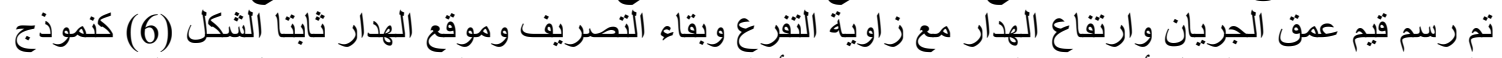

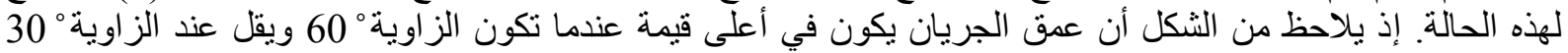

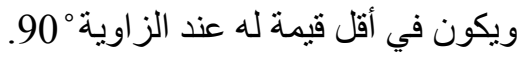

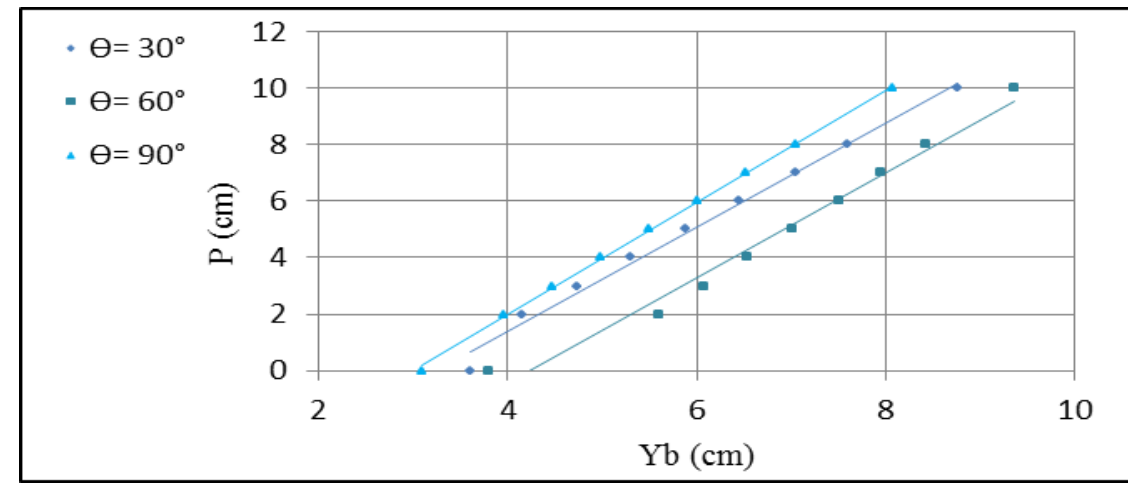

الثكل(6): العلاقة بين عمق الجريان الفرعي وارتفاع الهدار (X=60cm, QT = 17.25 l/sec).

الاستنتاجات

1. تزدداد نسبة التصريف الفرعي Qr (التصريف الفرعي مقسوما على التصريف الكلي) بزيادة ارتفاع الهدار مع وجود

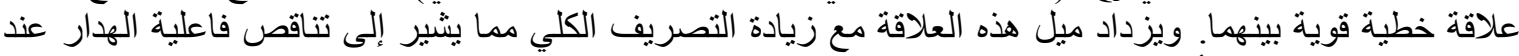

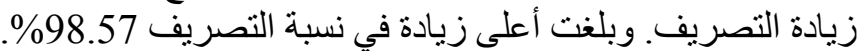
2. الزيادة في نسبة التصريف الفرعي تقل على على الأكثر مع زيادة التصريف عند ثبات بقية المتغيرات الأخرى (ارتفاع

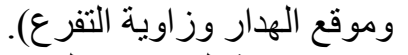

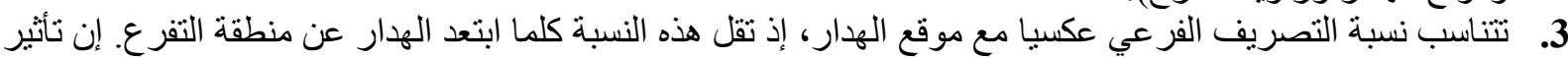

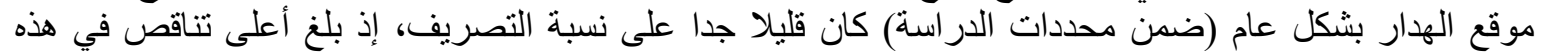

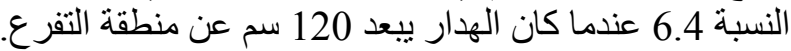

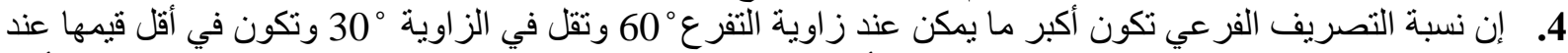

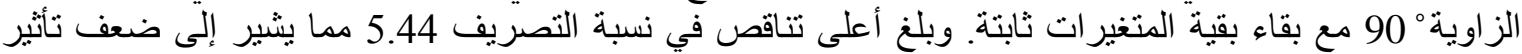
زاوية التفرع في هذه الدر اسة. 5. ينأثر عمق الماء في القناة الفرعية طرديا مع ارتفاع الهدار في القناة الرئيسة، إذ بلغت أعلى نسبة للزيادة في عمق الماء $\% 194$

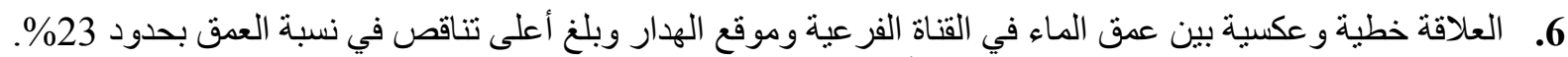
7. 


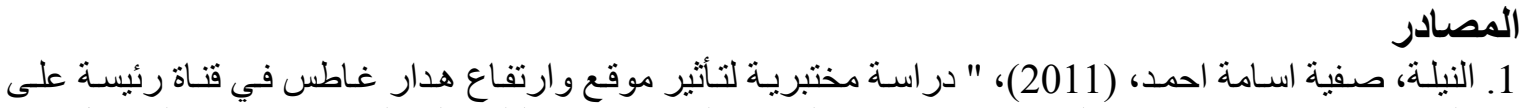

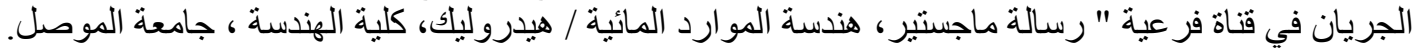

2. Ghostine, R., Mose, R., Vazquez, J., Ghenain, A., and Gregoire, C., (2010), "TwoDimensional Simulation of Subcritical Flow at a Combining Junction: Luxury or Necessity?", ASCE, J. of Hydraulic Engineering, Vol. 136, No.10, pp. 799-805.

3. Gurram, S. K. (1994). "A Study of Subcritical and Transitional Combining Flow in Open Channel Junction." Ph. D. thesis, Banaras Hindu

Univ., Varanasi, India,.

4. Hsu, C. C., Lee W. J., and Chang, C. H. (1998a). " Subcritical Open Channel Junction Flow.” J. Hydraul. Eng., 124(8), 847- 855.

5. Kesserwani, G., Vazquez, J., Riviere, N., Liang, Q., Travin, G., and Mose, R., (2010), "New Approach for Predicting Flow Bifurcation at Right- Angled Open- Channel Junction", ASCE, J. of Hydraulic Engineering, Vol. 136,No.9, pp. 662-668.

6. Neary, V.S. and Odgaard, A.J., (1993), “Three-Dimensional Flow Structure at OpenChannel Diversion”, ASCE, J. of Hydraulic Engineering, Vol. 119,No.11, pp. 1223-1230.

7. Ramamurthy, A.S., Qu, J., and Zhai, C., (2006), “3D Simulation of Combining Flow in $90^{\circ}$ Rectangular Closed Conduits", ASCE, J. of Hydraulic Engineering, Vol. 132,No. 2, pp. 214218.

8. Riviere, N., Travian, G., and Perkins, R. J. (2007). “ Transcritical Flows in Open Channel Intersection." Proc., 32 ${ }^{\text {nd }}$ IAHR Congress (CD-ROM), Venice, Italy, IAHR, Paper SS05- 11.

9. Shabayek, S., Seffler, P., and Hicks, F. (2002). “ Dynamic Model for Subcritical Combining Flows in Channel Junctions.” J. Hydraul. Eng., 128(9), 821- 828.

10. Taylor, E. H. (1944). " Flow Characteristics at Rectangular Open- Channel Junctions." Trans. Am. Soc. Civ. Eng., 109, 893- 912.

11. Webber, N. B., and Greated, C. A. (1966). "An Invastigation of Flow Behavior at the Junction of Rectangular Channels." Proc., Institute Civil Engineers, Vol. 34, London, pp. 321334, 321- 334.

$$
\text { تم اجراء البحث في كلية ألهنسسة = جامعة ألموصل }
$$

